\title{
TCEC Cup 6
}

Article

Accepted Version

TCEC Cup 6 report

Haworth, G. and Hernandez, N. (2020) TCEC Cup 6. ICGA Journal, 42 (2-3). pp. 237-241. ISSN 1389-6911 doi: https://doi.org/10.3233/ICG-200159 Available at https://centaur.reading.ac.uk/91840/

It is advisable to refer to the publisher's version if you intend to cite from the work. See Guidance on citing.

Published version at: https://content.iospress.com/articles/icga-journal/icg200159

To link to this article DOI: http://dx.doi.org/10.3233/ICG-200159

Publisher: The International Computer Games Association

All outputs in CentAUR are protected by Intellectual Property Rights law, including copyright law. Copyright and IPR is retained by the creators or other copyright holders. Terms and conditions for use of this material are defined in the End User Agreement.

\section{www.reading.ac.uk/centaur}

\section{CentAUR}

Central Archive at the University of Reading

Reading's research outputs online 


\section{TCEC Cup 6}

Guy Haworth and Nelson Hernandez ${ }^{1}$

Reading, UK and Maryland, USA

The TCEC Cup 6 knockout event was the last of TCEC Season 18, began on July $17^{\text {th }} 2020$ with the usual brisk 30' $+5^{\prime \prime}$ Rapid tempo. It involved the top 32 engines of the TCEC18 championship and used the rules of TCEC Cup 5 (CPW, 2020; Haworth and Hernandez, 2020a/b). Matches were 'best of four' and tie-breaks consisted of further 'same opening' mini-matches of two games.

This time, a different 'equal distance' pairing was used, with playing seed $s+2^{5-r}$ (rather than $2^{6-r}-s+1$ ) in round $r$ if the wins all go to the higher seed. Thus, seed $s 1$ plays $s 17, s 9, \ldots, s 2$ if all survive long enough. The higher seed is listed first in Table 1. This pairing also adheres to the Postponement Principle of keeping top seeds apart but stiffens the competition for the top quarter of the seeding and reduces the likelihood of protracting matches far into a tie-break - at least, in the early rounds. Here, seed $s$ is not sentient and therefore not in a position to wish it was seed $s+1$ !

The second author here allocated openings of $4,8,12$ and 16 ply to the first four rounds: Jeroen Noomen's openings for the finals came from his Superfinal books for TCEC seasons 9-18. Both chose randomly with some regard for frequency over the board providing the usual variety of play.

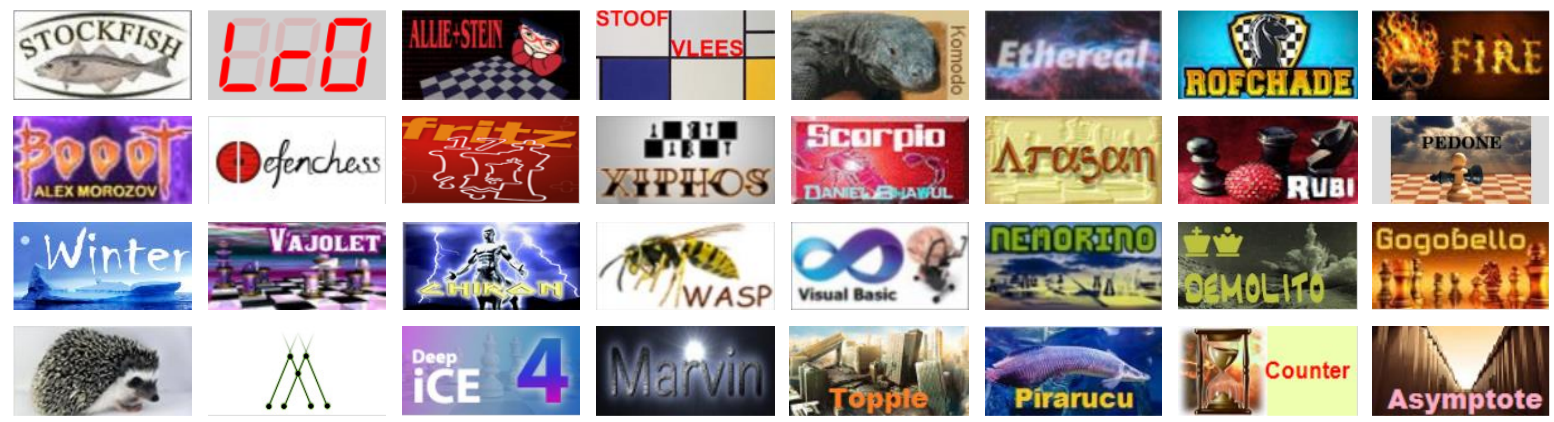

Fig. 1. Logos for TCEC Cup engines in seeded order (StOCKFISH $\rightarrow$ LEELA Chess Zero $\rightarrow \ldots \rightarrow$ AsymPTOTE).

As in previous TCEC Cup events, interest focused on engine's actual performance $\% P$ compared with their expected performance $E \% P$ implied by TCEC Elo difference Elo $\Delta$. How long will it be before STOCKFISH concedes a half-point?! The accuracy of the TCEC Elos is always a matter for debate, especially for the newer engines, and TCEC was about to appraise these relative to CCRL ratings. The upgrades to some eighteen of the engines - 'kudos' to their authors - and the influence of the random openings (even when games are repeated with colours flipped) are two other factors which can affect the match results.

\footnotetext{
${ }^{1}$ Corresponding author: g.haworth@ reading.ac.uk
} 
Table 1. TCEC Cup 6: round one results from the winner's perspective. ${ }^{2}$

\begin{tabular}{|c|c|c|c|c|c|c|c|c|c|c|c|c|}
\hline$\alpha \beta$ & $\#$ & $\stackrel{3}{g}$ & $\begin{array}{c}\text { Elo } \\
\Delta\end{array}$ & Elo & $\stackrel{\Xi}{\Xi}$ & छัँ & Round 1 Pairings & E\%P & Round 1 Results & $\# \mathbf{g}$ & $\% \mathbf{P}$ & par.. \\
\hline $\begin{array}{l}\text { St } \\
\text { Wi }\end{array}$ & 01 & $\begin{array}{l}\checkmark \\
\checkmark\end{array}$ & 222 & $\begin{array}{l}3551 \\
3329\end{array}$ & $\begin{array}{l}\mathrm{P} \\
2\end{array}$ & $\begin{array}{l}01 \\
17\end{array}$ & $\begin{array}{l}\text { Stockfish } 2020071122 \\
\text { Winter } 0.8 .4 \mathrm{a}\end{array}$ & 78.14 & Stockfish, 3-0: 111 & 3 & 100.00 & + \\
\hline $\begin{array}{l}\text { Bo } \\
\text { Ig }\end{array}$ & 02 & $\begin{array}{l}x \\
\checkmark\end{array}$ & 144 & $\begin{array}{l}3424 \\
3280\end{array}$ & $\begin{array}{l}1 \\
3\end{array}$ & & $\begin{array}{c}\text { Booot } 6.4 \\
\text { Igel 2.6-dev-3 }\end{array}$ & \} 69.29 & Igel, 21/2-1/2: $1=1$ & 3 & 16.67 & --- \\
\hline $\begin{array}{l}\mathrm{Ko} \\
\mathrm{CB}\end{array}$ & 03 & $\begin{array}{l}\checkmark \\
\times\end{array}$ & 211 & $\begin{array}{l}3509 \\
3298\end{array}$ & $\begin{array}{l}\mathrm{P} \\
2\end{array}$ & & $\begin{array}{l}\text { Komodo } 2570.00 \\
\text { ChessBrainVB }\end{array}$ & \} 76.99 & Komodo, 21/2-1/2: $1=1$ & 3 & 83.33 & $=$ \\
\hline $\begin{array}{l}\text { Sc } \\
\text { To }\end{array}$ & 04 & $\begin{array}{l}\checkmark \\
\checkmark\end{array}$ & 240 & $\begin{array}{l}3447 \\
3207\end{array}$ & $\begin{array}{l}1 \\
3\end{array}$ & $\begin{array}{l}13 \\
29\end{array}$ & $\begin{array}{c}\text { ScorpioNN 3.0.8.3 } \\
\text { Topple 0.7.5-20200605 }\end{array}$ & 79.95 & ScorpioNN, 3-0: 111 & 3 & 100.00 & + \\
\hline $\begin{array}{l}\mathrm{AS} \\
\mathrm{Ch}\end{array}$ & 05 & $\begin{array}{l}* \\
\times\end{array}$ & 245 & $\begin{array}{l}3537 \\
3292\end{array}$ & $\begin{array}{l}\mathrm{P} \\
2\end{array}$ & $\begin{array}{l}03 \\
19\end{array}$ & $\begin{array}{c}\text { AllieStein v0.7_dev2-net_15.0 } \\
\text { Chiron TCEC16 }\end{array}$ & 80.44 & AllieStein, 3-0: 111 & 3 & 100.00 & + \\
\hline $\begin{array}{l}\mathrm{Fr} \\
\mathrm{iC}\end{array}$ & 06 & $\begin{array}{l}x \\
x\end{array}$ & 129 & $\begin{array}{l}3384 \\
3255\end{array}$ & $\begin{array}{l}1 \\
3\end{array}$ & $\begin{array}{l}11 \\
27\end{array}$ & $\begin{array}{c}\text { Fritz } 17 \_20200130 \\
\text { iCE } 4.0 .853\end{array}$ & 67.42 & Fritz, 3-0: 111 & 3 & 100.00 & + \\
\hline $\begin{array}{c}\mathrm{rf} \\
\mathrm{Dm}\end{array}$ & 07 & $\begin{array}{l}\checkmark \\
\checkmark\end{array}$ & 138 & $\begin{array}{l}3434 \\
3296\end{array}$ & $\begin{array}{l}1 \\
3\end{array}$ & $\begin{array}{l}07 \\
23\end{array}$ & $\begin{array}{c}\text { rofChade } 2.305 \\
\text { Demolito } 20200711\end{array}$ & 68.55 & rofChade 3-1: ==11 & 4 & 75.00 & + \\
\hline $\begin{array}{l}\mathrm{Ru} \\
\mathrm{Co}\end{array}$ & 08 & $\begin{array}{l}\checkmark \\
\checkmark\end{array}$ & 243 & $\begin{array}{l}3402 \\
3159\end{array}$ & $\begin{array}{l}2 \\
Q\end{array}$ & & $\begin{array}{c}\text { RubiChess 1.8-dev_TCEC_Cup } \\
\text { Counter 3.6dev }\end{array}$ & 80.25 & Rubichess, $2^{1 / 2-1 / 2:} 1=1$ & 3 & 83.33 & $=$ \\
\hline $\begin{array}{l}\mathrm{Lc} \\
\mathrm{Va}\end{array}$ & 09 & $\begin{array}{l}\checkmark \\
\times\end{array}$ & 186 & $\begin{array}{l}3553 \\
3367\end{array}$ & $\begin{array}{l}\mathrm{P} \\
2\end{array}$ & $\begin{array}{l}02 \mathrm{~L} \\
18\end{array}$ & $\begin{array}{l}\text { CZero v0.26.0_sv-t60-4229-mlh_opt2 } \\
\text { Vajolet2 2.9.0-TCEC-S17 }\end{array}$ & 74.25 & LCZero, $2^{1 / 2-1 / 2: 1=1}$ & 3 & 83.33 & + \\
\hline $\begin{array}{l}\mathrm{De} \\
\mathrm{Mi}\end{array}$ & 10 & $\stackrel{x}{s}$ & 170 & $\begin{array}{l}3420 \\
3250\end{array}$ & $\begin{array}{l}1 \\
3\end{array}$ & $\begin{array}{l}10 \\
26\end{array}$ & $\begin{array}{c}\text { Defenchess 2.3_dev2 } \\
\text { Minic } 2.40\end{array}$ & 72.41 & Defenchess, $2 \frac{1}{2}-1 \frac{1}{2}: 1===$ & 4 & 62.50 & - \\
\hline $\begin{array}{l}\mathrm{Et} \\
\mathrm{Ne}\end{array}$ & 11 & $\begin{array}{l}\checkmark \\
\checkmark\end{array}$ & 166 & $\begin{array}{l}3472 \\
3306\end{array}$ & $\begin{array}{l}\mathrm{P} \\
2\end{array}$ & $\begin{array}{l}06 \\
22\end{array}$ & $\begin{array}{l}\text { Ethereal } 12.28 \\
\text { Nemorino } 5.40\end{array}$ & 71.94 & Ethereal, $2^{1 / 2-1 / 2: 2} 1=1$ & 3 & 83.33 & + \\
\hline $\begin{array}{l}\mathrm{Ar} \\
\mathrm{Pi}\end{array}$ & 12 & $\begin{array}{l}\checkmark \\
\times\end{array}$ & 165 & $\begin{array}{l}3390 \\
3225\end{array}$ & $\begin{array}{l}1 \\
3\end{array}$ & $\begin{array}{l}14 \\
30\end{array}$ & $\begin{array}{l}\text { Arasan 22.1_94foc64 } \\
\quad \text { Pirarucu 3.3.5 }\end{array}$ & 71.82 & Arasan, $2^{1 / 2-1 / 2: 11=}$ & 3 & 83.33 & + \\
\hline $\begin{array}{l}\mathrm{Sv} \\
\mathrm{Wa}\end{array}$ & 13 & $\stackrel{x}{s}$ & 175 & $\begin{array}{l}3510 \\
3335\end{array}$ & $\begin{array}{l}\mathrm{P} \\
2\end{array}$ & $\begin{array}{l}04 \\
20\end{array}$ & $\begin{array}{l}\text { Stoofvlees II a14 } \\
\text { Wasp } 4.01\end{array}$ & 72.99 & Stoofvlees, $2^{1 / 2-1 / 2: 1=1}$ & 3 & 83.33 & + \\
\hline $\begin{array}{c}\mathrm{Xi} \\
\mathrm{Ma}\end{array}$ & 14 & $\stackrel{x}{x}$ & 234 & $\begin{array}{l}3456 \\
3222\end{array}$ & $\begin{array}{l}1 \\
3\end{array}$ & $\begin{array}{l}12 \\
28\end{array}$ & $\begin{array}{c}\text { Xiphos 0.6.1 } \\
\text { Marvin 4.0.0-a7 }\end{array}$ & 79.36 & Xiphos, 4-2: =====, 11 & 6 & 66.67 & -- \\
\hline $\begin{array}{l}\mathrm{Fi} \\
\mathrm{Go}\end{array}$ & 15 & $\begin{array}{l}x \\
x\end{array}$ & 236 & $\begin{array}{l}3479 \\
3243\end{array}$ & $\begin{array}{l}1 \\
3\end{array}$ & $\begin{array}{l}08 \\
24\end{array}$ & $\begin{array}{c}\text { Fire } 8 \_ \text {beta } \\
\text { Gogobello } 2.2\end{array}$ & \} 79.56 & Fire, 3-0: 111 & 3 & 100.00 & + \\
\hline $\begin{array}{l}\mathrm{Pe} \\
\mathrm{At}\end{array}$ & 16 & $x$ & 212 & $\begin{array}{l}3351 \\
3139\end{array}$ & $\begin{array}{l}2 \\
\mathrm{Q}\end{array}$ & $\begin{array}{l}16 \\
32\end{array}$ & $\begin{array}{l}\text { Pedone } 20200510 \\
\text { Asy mptote } 0.9-\mathrm{dev}\end{array}$ & 77.10 & Pedone, $2^{1 / 2-1 / 2: 2} 1=1$ & 3 & 83.33 & $=$ \\
\hline
\end{tabular}

STOCKFISH began proceedings as the current TCEC Cup holder with a quick 3-0 victory over WINTER. Notably, WINTER took STOCKFISH to a 6-man RP-BP 'mate in 42 moves' in the first game which is certainly difficult to win without endgame tables, see Fig. 2a. SCORPIONN, ALLIESTEIN, FRITZ and FIRE also achieved the whitewash. Usually, winners look better than they are because they have White first: ' $3-1$ ' would be a cosmetic improvement on ' $2 \frac{1}{2} 2^{-1 / 2}$ ' from the loser's point of view. In match 2 , an improved IGEL achieved the only cupset, notably beating BOOOT as Black in a French and then being gifted a technical default.

The new 'equal distance' pairing scheme resulted in only one match going to a tiebreak because Elo $\Delta$ was more narrowly grouped around 195. The midfield seeds were not matched against each other as before and no match-winner lost a game. A refreshed MARVIN put up stiff resistance but succumbed to XIPHOS, losing both sides of an inoffensive-sounding Giuoco Pianissimo.

\footnotetext{
${ }^{2}$ In these tables, the first-named engine played White first except where indicated by a '*'. Alongside the ' $\% \mathrm{P}$ ' column, '+' ('-') indicates an unexpected excess (shortfall) of a 1/2-point in the first engine's score. ' $\# \mathrm{~g}$ ' $\equiv$ number of games.
} 
Round 2

\begin{tabular}{|c|c|c|c|c|c|c|c|c|c|c|c|c|}
\hline$\alpha \beta$ & $\#$ & $=$ & $\begin{array}{c}\text { Elo } \\
\Delta\end{array}$ & Elo & 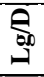 & 芯 & Round 2 Pairings & $\mathbf{E \%} \mathbf{P}$ & Round 2 Results & $\# \mathbf{g}$ & $\% \mathbf{P}$ & par.. \\
\hline $\begin{array}{l}\text { St } \\
\text { Ig }\end{array}$ & 01 & $\begin{array}{l}\checkmark \\
\checkmark\end{array}$ & 271 & $\begin{array}{l}3551 \\
3280\end{array}$ & $\begin{array}{l}\mathrm{P} \\
3\end{array}$ & $\begin{array}{l}01 \\
25\end{array}$ & $\begin{array}{c}\text { Stockfish } 2020071122 \\
\text { Igel 2.6-dev-3 }\end{array}$ & 82.86 & Stockfish, $2^{1 / 2-11 / 2:==1=}$ & 4 & 62.50 & -- \\
\hline $\begin{array}{l}\mathrm{Ko} \\
\mathrm{Sc}\end{array}$ & 02 & $\begin{array}{l}\checkmark \\
\checkmark\end{array}$ & 62 & $\begin{array}{l}3509 \\
3447\end{array}$ & $\begin{array}{c}\mathrm{P} \\
1\end{array}$ & $\begin{array}{l}05 \\
13\end{array}$ & $\begin{array}{c}\text { Komodo 2570.00 } \\
\text { ScorpioNN 3.0.8.3 }\end{array}$ & 58.59 & Komodo, 21/2-11/2: $101=$ & 4 & 62.50 & $=$ \\
\hline $\begin{array}{l}\text { AS } \\
\text { Fr }\end{array}$ & 03 & $\begin{array}{l}x \\
\times\end{array}$ & 153 & $\begin{array}{l}3537 \\
3384\end{array}$ & $\begin{array}{l}\mathrm{P} \\
1\end{array}$ & $\begin{array}{l}03 \\
11\end{array}$ & $\begin{array}{l}\text { AllieStein v0.7_dev2-net_15.0 } \\
\text { Fritz 17_20200130 }\end{array}$ & 70.38 & AllieStein, $2 \frac{1}{2}-1 \frac{1}{2} 2:==1=$ & 4 & 62.50 & - \\
\hline $\begin{array}{c}\mathrm{rf} \\
\mathrm{Ru}\end{array}$ & 04 & $\begin{array}{l}\checkmark \\
\checkmark\end{array}$ & 32 & $\begin{array}{l}3434 \\
3402\end{array}$ & $\begin{array}{l}1 \\
2\end{array}$ & $\begin{array}{l}07 \\
15\end{array}$ & $\begin{array}{c}\text { rofChade } 2.305 \\
\text { RubiChess } 1.8-\text { dev_TCEC_Cup }\end{array}$ & 54.46 & rofChade, $2 \frac{1}{2} 2-1 \frac{1}{2}:==1=$ & 4 & 62.50 & + \\
\hline $\begin{array}{l}\text { Lc } \\
\text { De }\end{array}$ & 05 & $\begin{array}{l}\checkmark \\
\times\end{array}$ & 133 & $\begin{array}{l}3553 \\
3420\end{array}$ & $\begin{array}{c}\mathrm{P} \\
1\end{array}$ & $\begin{array}{l}02 \\
10\end{array}$ & $\begin{array}{c}\text { CZero v0.26.0_sv-t60-4229-mlh_opt2 } \\
\text { Defenchess } 2.3 \text { _dev2 }\end{array}$ & 67.92 & LCZero, $2 \frac{1 / 2-1 / 2:=11}{1}$ & 3 & 83.33 & + \\
\hline $\begin{array}{l}\mathrm{Et} \\
\mathrm{Ar}\end{array}$ & 06 & $\begin{array}{l}\checkmark \\
\checkmark\end{array}$ & 82 & $\begin{array}{l}3472 \\
3390\end{array}$ & $\begin{array}{c}\mathrm{P} \\
1\end{array}$ & $\begin{array}{l}06 \\
14\end{array}$ & $\begin{array}{c}\text { Ethereal 12.28 } \\
\text { Arasan 22.1_94f0c64 }\end{array}$ & 61.29 & Ethereal, $2 \frac{1}{2}-1 / 2 / 2:=11$ & 3 & 83.33 & + \\
\hline $\begin{array}{l}\mathrm{Sv} \\
\mathrm{Xi}\end{array}$ & 07 & $\begin{array}{l}\times \\
\times\end{array}$ & 54 & $\begin{array}{l}3510 \\
3456\end{array}$ & $\begin{array}{c}\mathrm{P} \\
1\end{array}$ & $\begin{array}{l}04 \\
12\end{array}$ & $\begin{array}{c}\text { Stoofvlees II a14 } \\
\text { Xiphos 0.6.1 }\end{array}$ & 57.50 & Xiphos, $2 \frac{1}{2}-1^{1} \frac{1}{2}$ : $=1==$ & 4 & 37.50 & -- \\
\hline $\begin{array}{l}\mathrm{Fi} \\
\mathrm{Pe}\end{array}$ & 08 & $\begin{array}{l}\times \\
\times\end{array}$ & 128 & $\begin{array}{l}3479 \\
3351\end{array}$ & $\begin{array}{l}1 \\
2\end{array}$ & $\begin{array}{l}08 \\
16\end{array}$ & $\begin{array}{c}\text { Fire 8_beta } \\
\text { Pedone 20200510 }\end{array}$ & 67.29 & Fire, $21 / 2-1 \frac{1}{2}: 1===$ & 4 & 62.50 & $=$ \\
\hline
\end{tabular}

Table 2. TCEC Cup 6: round two results from the winner's perspective.

Here, average Elo $\Delta$ narrowed to 114 so we expected closer contests. In the first match, it was clear that IGEL had grown a full set of adult spines: it drew its first two games against STOCKFISH. However, it took its Queen out of the play by accepting a 'poisoned' rook, see Fig. 2b, and went down in the third game: STOCKFISH did not make the same mistake in the return. Watch out for IGEL in TCEC Season 19: this was a much better result than we were entitled to expect. KOMODO prevailed by the same score but not before SCORPIONN had the distinction of beating the eventual match winner.

The second cupset of the event was STOOFVLEES' loss to XIPHOS. We put it that way as STOOFVLEES blundered in game 2 as only it can. Fig. 2c's 49...h2??, optically but not tactically sound, was the start of a misconceived plan which rapidly unravelled on contact with the enemy.

\section{The quarterfinals, semi-finals, Bronze final and final}

Table 3. TCEC Cup 6: quarterfinal results from the winner's perspective.

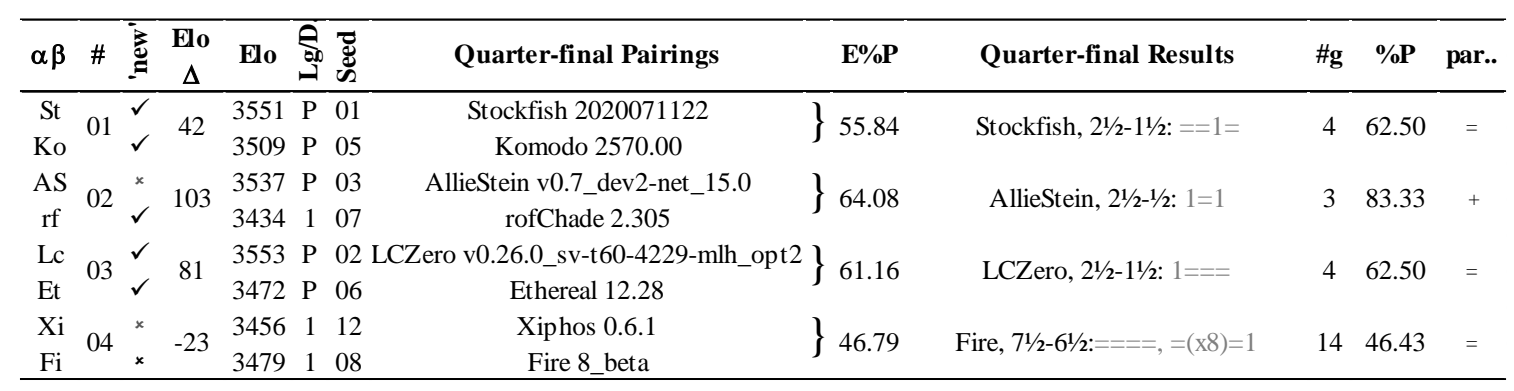

STOOFVLEES' demise at the hands of XIPHOS produced a predictably close 'Division 1' clash in the last match. In game 25 by move 45, XIPHOS thought it was lost before FIRE did. The other three quarterfinals went very much as expected, one win being decisive in each case. 
Table 4. TCEC Cup 6: semi-final results from the winner's perspective.

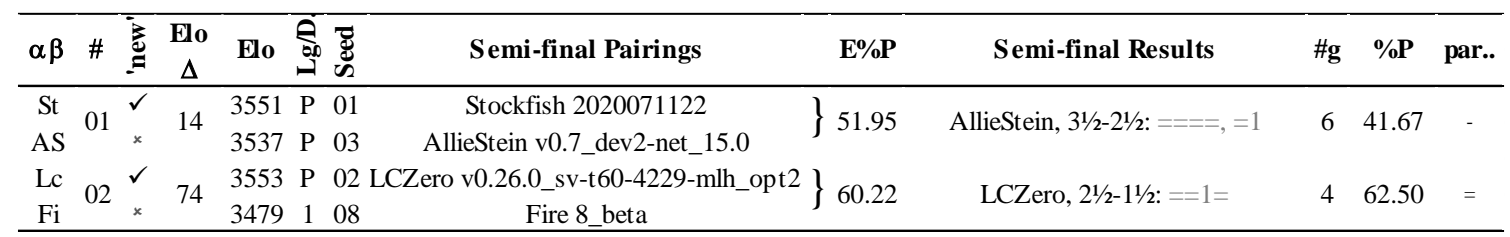
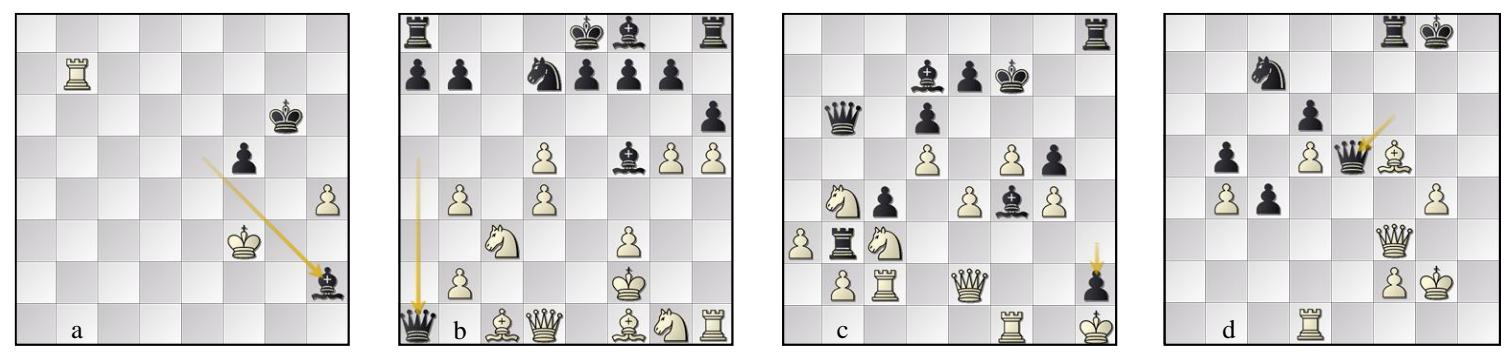

Fig. 2. (a) r1-g01 ST-Wi pos. 64w, (b) r2-g03 ST-Ig p15w, (c) r2-g24 XI-Sv p50w, (d) SF-g06 As-ST p47w.

The first semi-final went to extra time: no surprise. The evaluation graph for ALLIESTEIN's game 6 win, Fig. 4a, suggests that STOCKFISH never had a grip on the game, that maybe it lost its way around move 26 and that ALLIESTEIN was confident by move 34. By move 47, the victory via two connected passed pawns on the kingside was clear to both sides, see Fig. 2d: STOCKFISH saw it first.

In the play-off for the bronze medal, STOCKFISH unexpectedly met FIRE - there is irony in that. The games went as expected. FIRE gave a good account of itself as White and reached a drawn KQPKQ endgame on the attacking side in game 2, see Fig. 3b. However, it put up little resistance as Black and in both games, STOCKFISH thought it was ' +2 ' after 27 moves, see Figs. $3 \mathrm{a}$ and $3 \mathrm{c}$ and the evaluation graphs in Figs. 4b and 4c.

Table 5. TCEC Cup 6: the Stockfish - Fire play-off and the LeEla Chess Zero - AlLieStein final.

\begin{tabular}{|c|c|c|c|c|c|c|c|c|c|c|c|}
\hline$\alpha \beta$ & $\#$ & $\stackrel{3}{=}$ & $\begin{array}{c}\text { Elo } \\
\Delta\end{array}$ & Elo & 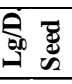 & Bronze Final \& Final Pairings & E\%P & Results & $\# \mathrm{~g}$ & $\% \mathbf{P}$ & par.. \\
\hline $\begin{array}{l}\mathrm{St} \\
\mathrm{Fi}\end{array}$ & 02 & $\begin{array}{l}\checkmark \checkmark \\
\times\end{array}$ & 72 & $\begin{array}{l}3551 \\
3479\end{array}$ & $\begin{array}{ll}\mathrm{P} & 01 \\
1 & 08\end{array}$ & $\begin{array}{l}\text { Stockfish 2020071122 } \\
\text { Fire 8_beta }\end{array}$ & \} 59.95 & Stockfish, 21/2-1/2: $1=1$ & 3 & 83.33 & + \\
\hline $\begin{array}{l}\text { AS } \\
\text { Lc }\end{array}$ & 01 & $\begin{array}{l}x \\
\checkmark\end{array}$ & -16 & $\begin{array}{l}3537 \\
3553\end{array}$ & $\begin{array}{ll}\mathrm{P} & 03 \\
\mathrm{P} & 02\end{array}$ & $\begin{array}{l}\text { AllieStein v0.7_dev2-net_15.0 } \\
\text { Zero v0.26.0_sv-t60-4229-mlh_opt }\end{array}$ & \} 47.77 & AllieStein, $2 \frac{1}{2}-1 \frac{1}{2} 2: 1===$ & 4 & 62.50 & + \\
\hline
\end{tabular}
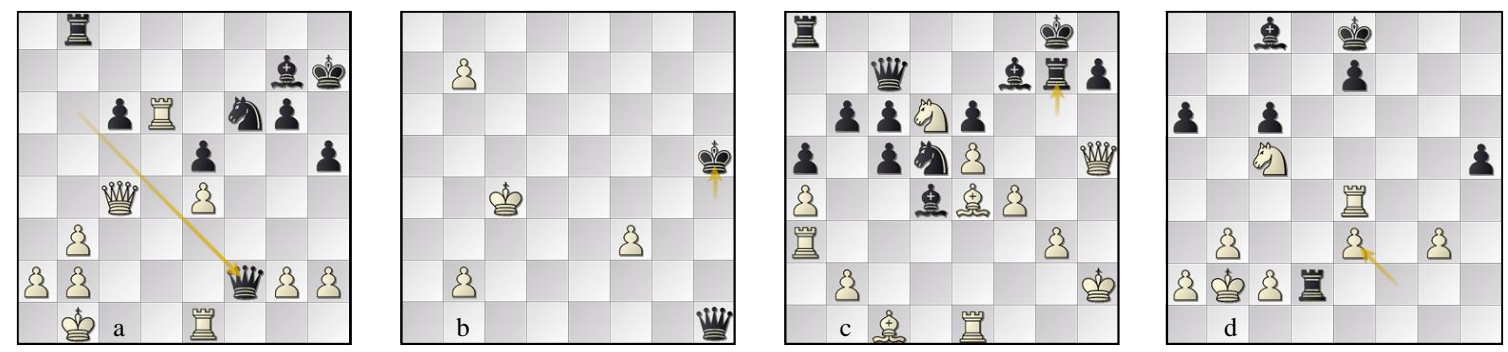

Fig. 3. (a) BF-g01 ST-Fi p28w, (b) BF-g02 Fi-ST p44w, (c) BF-g03 St-Fi p28w, (d) F-g01 As-Lc p37b. 

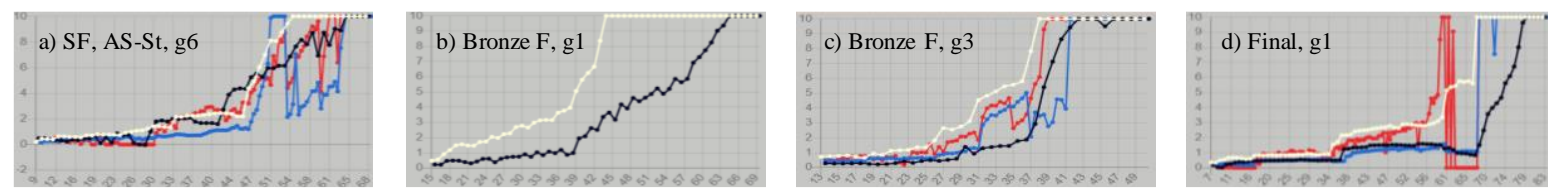

Fig. 4. (a) Semi Final, AS-St game 5; Bronze F., ST-Fi (b) g1 and (c) g3; (d) Final, AS-Lc g1.

The first TCEC final between two neural network engines was surprisingly brief: ALLIESTEIN won the first game, Fig. 4d, and this was enough. AllieStein saw an opportunity to dominate queenside after 34...Rd2 and gain a crucial pawn advantage in a rook endgame. The endgame tables must have driven the final evaluations as the KRPPkrp mate was still 30 moves away at adjudication time.

\section{$4 \quad$ In conclusion}

TCEC's Cup 6 knockout event was another brisk and enjoyable celebration of chess. We congratulate ALLIESTEIN's authors and trainers on winning the TCEC Cup on this sixth occasion, their first TCEC title. ALLIESTEIN faced STOCKFISH in the TCEC16 Superfinal and will be well fancied for the TCEC Championship in season 19.

Thanks also go to all engine authors for including their creations again, the majority in new versions. The standard of play at Rapid tempo is remarkably high and all games, somewhat annotated are available (Haworth and Hernandez, 2020c). Finally, with the global pandemic still unbeaten, we thank all TCEC's volunteers and connections for their excellent work in putting on Season 18.

Table 6. The shortest and longest 1-0, drawn and 0-1 games in each phase of TCEC Cup 6:

' $48 / 1$ ' in row 1, column 2 for example means 'game 48 in the pgn, game 1 in the match'.

\begin{tabular}{|c|c|c|c|c|c|c|c|c|c|c|c|c|c|c|c|c|c|c|}
\hline \multirow{4}{*}{$\begin{array}{c}\text { Round } \\
1\end{array}$} & \multicolumn{6}{|c|}{ 1-0 } & \multicolumn{6}{|c|}{$1 / 2-1 / 2$} & \multicolumn{6}{|c|}{$0-1$} \\
\hline & \multicolumn{3}{|c|}{ Shortest } & \multicolumn{3}{|c|}{ Longest } & \multicolumn{3}{|c|}{ Shortest } & \multicolumn{3}{|c|}{ Longest } & \multicolumn{3}{|c|}{ Shortest } & \multicolumn{3}{|c|}{ Longest } \\
\hline & \multicolumn{2}{|c|}{ Game } & \multirow{2}{*}{$\begin{array}{c}\# \mathbf{m v} \\
33\end{array}$} & \multicolumn{2}{|c|}{ Game } & \multirow{2}{*}{$\begin{array}{c}\# \mathbf{m v} \\
99\end{array}$} & \multicolumn{2}{|c|}{ Game } & \multirow{2}{*}{$\begin{array}{c}\# \mathbf{m v} \\
25\end{array}$} & \multicolumn{2}{|c|}{ Game } & \multirow{2}{*}{$\begin{array}{c}\text { \#mv } \\
67\end{array}$} & \multicolumn{2}{|c|}{ Game } & \multirow{2}{*}{$\begin{array}{c}\# \mathbf{m v} \\
42\end{array}$} & \multicolumn{2}{|c|}{ Game } & \multirow{2}{*}{$\begin{array}{c}\text { \#mv } \\
93\end{array}$} \\
\hline & $48 / 1$ & Fi-Go & & $10 / 1$ & Sc-To & & $5 / 2$ & Ig-Bo & & $32 / 4$ & Mi-De & & $49 / 2$ & Go-Fi & & $14 / 2$ & & \\
\hline 2 & $3 / 3$ & & 39 & $6 /$ & & 88 & $29 / 3$ & & & $20 / 1$ & & 125 & $21 / 2$ & $\mathrm{Ar}-\mathrm{Et}$ & & $18 / 2$ & De-Lc & 72 \\
\hline QF & $3 / 3$ & St-Ko & 63 & $8 / 1$ & Lc-Et & 180 & $18 / 7$ & $\mathrm{Xi}-\mathrm{Fi}$ & 39 & $16 / 5$ & $\mathrm{Xi}-\mathrm{Fi}$ & 136 & - & - & - & - & - & - \\
\hline SF & $6 / 6$ & AS-St & 68 & $9 / 3$ & $\mathrm{Lc}-\mathrm{Fi}$ & 79 & $3 / 3$ & St-AS & 46 & $5 / 5$ & St-AS & 57 & - & - & - & - & - & - \\
\hline Bronze F. & 3 & St-Fi & 50 & 1 & $\mathrm{St}-\mathrm{Fi}$ & 69 & 2 & $\mathrm{Fi}-\mathrm{St}$ & 43 & 2 & $\mathrm{Fi}-\mathrm{St}$ & 43 & - & - & - & - & - & - \\
\hline Final & 1 & AS-Lc & 83 & 1 & AS-Lc & 83 & 2 & Lc-AS & 46 & 3 & AS-Lc & 122 & - & - & - & - & - & - \\
\hline Overall & 1,48 & Fi-Go & 33 & QF, 8 & Lc-Et & 180 & 1,5 & Ig-Bo & 25 & $\mathrm{QF}, 16$ & $\mathrm{Xi}-\mathrm{Fi}$ & 136 & 1,49 & Go-Fi & 42 & 1,14 & Ch-AS & 93 \\
\hline
\end{tabular}

\section{REFERENCES}

CPW (2020) https://tinyurl.com/icga046. Biographies of chess engines, authors and developers.

Haworth, G. M'C. and Hernandez, N. (2020a). TCEC Cup 5, ICGA Journal, 42(2-3), 207-210. http://centaur.reading.ac.uk/90606/ adds pgn files, further data and statistics.

Haworth, G. $M^{\mathrm{c}} \mathrm{C}$. and Hernandez, N. (2020b). TCEC18: the $18^{\text {th }}$ Top Chess Engine Championship, ICGA Journal, 42(2-3), 211-222. http://centaur.reading.ac.uk/91839/ adds pgn files, further data and statistics.

Haworth, G. $M^{\mathrm{c} C}$. and Hernandez (2020c). This report on TCEC Cup 6, ICGA Journal, 42(2-3), $237-$ 241. http://centaur.reading.ac.uk/91840/ adds pgn files, further data and statistics. 